\title{
EXTREMAL GRAPHS FOR A BOUND ON THE ROMAN DOMINATION NUMBER
}

\author{
Ahmed Bouchou $^{1}$, Mostafa Blidia $^{2}$ \\ AND \\ Mustapha Chellali ${ }^{2}$ \\ ${ }^{1}$ Department of Mathematics and Computer Science \\ University of Médéa, Algeria \\ ${ }^{2}$ LAMDA-RO Laboratory \\ Department of Mathematics \\ University of Blida B.P. 270, Blida, Algeria \\ e-mail: bouchou.ahmed@yahoo.fr \\ m_blidia@yahoo.fr \\ m_chellali@yahoo.com
}

\begin{abstract}
A Roman dominating function on a graph $G=(V, E)$ is a function $f: V(G) \longrightarrow\{0,1,2\}$ such that every vertex $u$ for which $f(u)=0$ is adjacent to at least one vertex $v$ with $f(v)=2$. The weight of a Roman dominating function is the value $w(f)=\sum_{u \in V(G)} f(u)$. The minimum weight of a Roman dominating function on a graph $G$ is called the Roman domination number of $G$, denoted by $\gamma_{R}(G)$. In 2009, Chambers, Kinnersley, Prince and West proved that for any graph $G$ with $n$ vertices and maximum degree $\Delta, \gamma_{R}(G) \leq n+1-\Delta$. In this paper, we give a characterization of graphs attaining the previous bound including trees, regular and semiregular graphs. Moreover, we prove that the problem of deciding whether $\gamma_{R}(G)=n+1-\Delta$ is co- $\mathcal{N} \mathcal{P}$-complete. Finally, we provide a characterization of extremal graphs of a Nordhaus-Gaddum bound for $\gamma_{R}(G)+\gamma_{R}(\bar{G})$, where $\bar{G}$ is the complement graph of $G$.
\end{abstract}

Keywords: Roman domination, Roman domination number, NordhausGaddum inequalities.

2010 Mathematics Subject Classification: 05C69. 
[1] S. Bermudo, H. Fernau and J. Sigarreta, The differential and the Roman domination number of a graph, Appl. Anal. Discrete Math. 8 (2014) 155-171.

doi:10.2298/AADM140210003B

[2] E.W. Chambers, B. Kinnersley, N. Prince and D.B. West, Extremal problems for Roman domination, SIAM J. Discrete Math. 23 (2009) 1575-1586. doi:10.1137/070699688

[3] E.J. Cockayne, P.A. Dreyer Jr., S.M. Hedetniemi and S.T. Hedetniemi, Roman domination in graphs, Discrete Math. 278 (2004) 11-22. doi:10.1016/j.disc.2003.06.004

[4] M.R. Garey and D.S. Johnson, Computers and Intractability: A Guide to the Theory of NP-completeness (Freeman, New York, 1979).

[5] W. McCuaig and B. Shepherd, Domination in graphs with minimum degree two, J. Graph Theory 13 (1989) 749-762. doi:10.1002/jgt.3190130610

[6] B.P. Mobaraky and S.M. Sheikholeslami, Bounds on Roman domination numbers of graphs, Mat. Vesnik 60 (2008) 247-253.

[7] C.S. ReVelle and K.E. Rosing, Defendens Imperium Romanum: a classical problem in military strategy, Amer. Math. Monthly 107 (2000) 585-594. doi:10.1080/00029890.2000.12005243

[8] I. Stewart, Defend the Roman Empire!, Sci. Amer. 281 (1999) 136-138. doi:10.1038/scientificamerican1299-136

Received 11 December 2017

Revised 24 April 2018 Accepted 24 April 2018 